AperTO - Archivio Istituzionale Open Access dell'Università di Torino

\title{
Monotone twist maps and periodic solutions of systems of Duffing type
}

\section{This is the author's manuscript}

Original Citation:

Availability:

This version is available http://hdl.handle.net/2318/151180

since

Published version:

DOI: $10.1017 / \mathrm{S} 0305004114000310$

Terms of use:

Open Access

Anyone can freely access the full text of works made available as "Open Access". Works made available under a Creative Commons license can be used according to the terms and conditions of said license. Use of all other works requires consent of the right holder (author or publisher) if not exempted from copyright protection by the applicable law. 


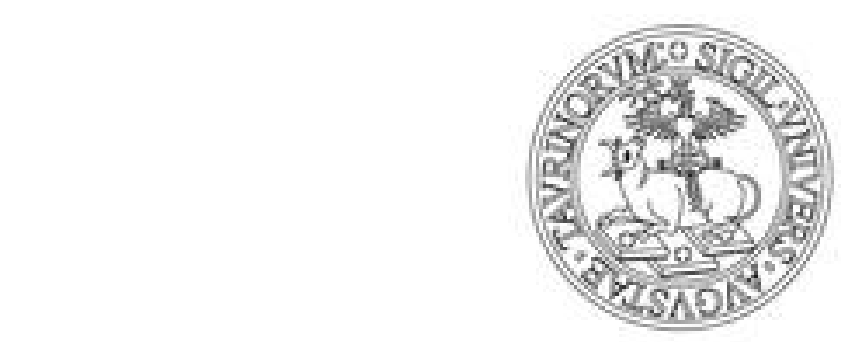

\section{UNIVERSITÀ DEGLI STUDI DI TORINO}

This is an author version of the contribution published on:

A. Boscaggin, R. Ortega

Monotone twist maps and periodic solutions of systems of Duffing type

MATHEMATICAL PROCEEDINGS OF THE CAMBRIDGE

PHILOSOPHICAL SOCIETY (2014) 157

DOI: $10.1017 / \mathrm{S} 0305004114000310$

The definitive version is available at:

http://www.journals.cambridge.org/abstract_S0305004114000310 


\title{
Monotone twist maps and periodic solutions of systems of Duffing type
}

\author{
By ALBERTO BOSCAGGIN \\ Dipartimento di Matematica, Università di Torino, \\ Via Carlo Alberto 10, I-10123 Torino, Italy \\ AND RAFAEL ORTEGA \\ Departamento de Matemática Aplicada, Universidad de Granada, \\ E-18071 Granada, Spain
}

(Received ?? May 2013; revised ?? April 2014)

\section{Abstract}

The theory of twist maps is applied to prove the existence of many harmonic and subharmonic solutions for certain Newtonian systems of differential equations. The method of proof leads to very precise information on the oscillatory properties of these solutions.

\section{Introduction}

In a series of papers which appeared between 1955 and 1976, G.R. Morris discovered many properties of the forced Duffing equation

$$
x^{\prime \prime}+2 x^{3}=p(t)
$$

with $p(t)$ continuous and $2 \pi$-periodic. In particular he proved in $[\mathbf{9}]$ the existence of infinitely many periodic solutions with least period $2 k \pi, k \geqslant 1$. In [9] it was also assumed that $p(t)$ is of class $C^{1}$ and has zero average but these conditions are inessential. To prove his result Morris considered the Poincare map $T$ associated to the $2 \pi$-periodic equation $(1 \cdot 1)$. This is an area-preserving homeomorphism of the plane and the periodic solutions of $(1 \cdot 1)$ are in correspondence with the fixed points of the iterates $T^{k}$. The essence of the proof is the construction of a family of Jordan curves $\mathcal{C}$ such that if $U=T^{k}$ then the points lying on $\mathcal{C} \cap U(\mathcal{C})$ are fixed under $U$. Each of these curves contains at least two fixed points of $T^{k}$ and acts as a label for periodic solutions, carrying information on their oscillatory behaviour. We recall that this additional information plays a crucial role to detect the minimality of the period when facing the problem of subharmonic solutions.

The reader who is familiar with the Poincaré-Birkhoff theorem will associate Morris' approach with the proof of this theorem in the particular case of monotone twist maps. We recall that a smooth map of the plane has monotone twist if it is expressed in polar coordinates $(\theta, \rho) \mapsto\left(\theta_{1}, \rho_{1}\right)$ and

$$
\frac{\partial \theta_{1}}{\partial \rho}>0
$$

See Section 2.8 of the book by Moser and Zehnder [10] for more details. 
The results in $[\mathbf{9}]$ have been extended in many ways. Typically these extensions employ sophisticated versions of the Poincaré-Birkhoff theorem and the condition of monotone twist is not required. In this direction we mention the paper by Ding and Zanolin [3] and the references therein.

To prove the existence of periodic solutions for systems it seems natural to look for extensions of the Poincaré-Birkhoff theorem to more degrees of freedom. This is a challenging question and our goal will be less ambitious. We intend to show that the more elementary approach using monotone twist maps can be extended to higher dimensions. Assume that $U$ is a diffeomorphism of $\mathbb{R}^{2 N}=\mathbb{R}^{2} \times \cdots \times \mathbb{R}^{2}$ that can be expressed in polar coordinates $U(\Theta, R)=\left(\Theta_{1}, R_{1}\right)$ with $\Theta=\left(\theta_{1}, \ldots, \theta_{N}\right)$ and $R=\left(\rho_{1}, \ldots, \rho_{N}\right)$. In addition assume that $U$ is symplectic, that is

$$
\sum_{i=1}^{N} d \theta_{i}^{1} \wedge d \rho_{i}^{1}=\sum_{i=1}^{N} d \theta_{i} \wedge d \rho_{i} .
$$

For $N=1$ this is equivalent to the area-preserving condition. For $N \geqslant 2$ this is a more restrictive condition. We say that $U$ satisfies the monotone twist condition if the symmetric matrix

$$
\partial_{R} \Theta_{1}+\left(\partial_{R} \Theta_{1}\right)^{t} \quad \text { is definite positive. }
$$

This assumption leads to an elementary version of the Poincaré-Birkhoff theorem that is valid in arbitrary dimension. All this is well known and we refer again to the book [10].

We will employ these ideas to prove the existence of many periodic solutions of the system

$$
x_{i}^{\prime \prime}+x_{i}^{3}=\frac{\partial P}{\partial x_{i}}\left(t, x_{1}, x_{2}\right), \quad i=1,2,
$$

where $P=P\left(t, x_{1}, x_{2}\right)$ is $2 \pi$-periodic in $t$ and has a second derivative vanishing at infinity. We will be more precise later on the conditions on $P$. By now we just present the model example $P=P_{0}$ with

$$
P_{0}\left(t, x_{1}, x_{2}\right)=\arctan \left(x_{1}\right) \arctan \left(x_{2}\right)+p_{1}(t) x_{1}+p_{2}(t) x_{2}
$$

with $p_{i}(t)$ continuous and $2 \pi$-periodic.

Variational methods are an alternative tool in the study of the periodic problem for $(1 \cdot 2)$. In [2] Bahri and Berestycki proved the existence of infinitely many periodic solutions for a system of the type

$$
x^{\prime \prime}+\nabla V(x)=p(t), \quad x \in \mathbb{R}^{N}
$$

when the potential satisfies the following condition: there exist numbers $R>0$ and $\theta \in] 0, \frac{1}{2}[$ such that

$$
V(x) \leqslant \theta\langle\nabla V(x), x\rangle \quad \text { if }|x| \geqslant R .
$$

This result is applicable to the previous example where $N=2$ and $V\left(x_{1}, x_{2}\right)=\frac{1}{4}\left(x_{1}^{4}+\right.$ $\left.x_{2}^{4}\right)-\arctan \left(x_{1}\right) \arctan \left(x_{2}\right)$. However it is not clear if the method in [2] carries the detailed information on the oscillatory properties of solutions that can be obtained via symplectic methods. More recently, Terracini and Verzini have employed a different variational technique in [12]. They obtain results on the existence of odd periodic solutions for certain systems in the class $(1 \cdot 2)$ and their method takes into account oscillatory properties. More work along these lines can be expected in the future. 
The rest of the paper is organized in two sections. In Section 2 we discuss some properties of maps that are exact symplectic and have monotone twist. We follow the presentation in [10] and adapt it for applications to systems of differential equations. In particular, we provide a version applicable to the iterates of a map. In applications it leads to a sharp counting of the subharmonic solutions. In Section 3 we state and prove the main result on (1.2). In particular we show that the iterates of the Poincaré map satisfy the monotone twist condition on large open sets close to infinity and we label periodic solutions in terms of their oscillatory behaviour. This will require careful estimates that seem to be new.

\section{Periodic points of maps with monotone twist}

In this section we present an abstract framework for the search of fixed points or periodic points. It is based on ideas taken from [10].

Consider the space $\mathbb{R}^{N} \times \mathbb{R}^{N}$ with coordinates $(\Theta, \mathcal{I})$ where $\Theta=\left(\theta_{1}, \ldots, \theta_{N}\right)$ and $\mathcal{I}=\left(I_{1}, \ldots, I_{N}\right)$. The coordinates $\theta_{i}$ will be called angles and the coordinates $I_{i}$ will be called actions. In agreement with this terminology we consider the torus

$$
\mathbb{T}^{N}=\mathbb{R}^{N} / 2 \pi \mathbb{Z}^{N}
$$

and the associated covering map

$$
\Pi: \mathbb{R}^{N} \times \mathbb{R}^{N} \rightarrow \mathbb{T}^{N} \times \mathbb{R}^{N}, \quad(\Theta, \mathcal{I}) \mapsto\left(\Theta+2 \pi \mathbb{Z}^{N}, \mathcal{I}\right) .
$$

Two points $\left(\Theta_{1}, \mathcal{I}_{1}\right)$ and $\left(\Theta_{2}, \mathcal{I}_{2}\right)$ are geometrically different if $\Pi\left(\Theta_{1}, \mathcal{I}_{1}\right) \neq \Pi\left(\Theta_{2}, \mathcal{I}_{2}\right)$. Given a region $\mathcal{D} \subset \mathbb{R}^{N}$ and $f=f(\Theta, \mathcal{I})$ defined on $\mathbb{R}^{N} \times \mathcal{D}$, we say that $f$ is $2 \pi$-periodic if

$$
f(\Theta+2 \pi \chi, \mathcal{I})=f(\Theta, \mathcal{I})
$$

for each $(\Theta, \mathcal{I}) \in \mathbb{R}^{N} \times \mathcal{D}$ and $\chi \in \mathbb{Z}^{N}$. Note that $f$ can be interpreted as a function defined on $\mathbb{T}^{N} \times \mathcal{D}$.

The symbols $\partial_{\Theta}$ and $\partial_{\mathcal{I}}$ will stand for the derivatives with respect to the variables $\Theta$ and $\mathcal{I}$, respectively. Typically these operators will act on functions taking values in $\mathbb{R}^{M}$, leading to Jacobian matrices with $M$ rows and $N$ columns. For simplicity vectors will be written as row vectors, but in computations involving matrices they must be thought as column vectors.

Let $\mathcal{D}$ be a convex and open subset of $\mathbb{R}^{N}$. We deal with a map $\Psi: \mathbb{R}^{N} \times \mathcal{D} \rightarrow \mathbb{R}^{N} \times \mathbb{R}^{N}$ of class $C^{1}$ such that, for every $(\Theta, \mathcal{I}) \in \mathbb{R}^{N} \times \mathcal{D}$ and $\chi \in \mathbb{Z}^{N}$,

$$
\Psi(\Theta+2 \pi \chi, \mathcal{I})=\Psi(\Theta, \mathcal{I})+(2 \pi \chi, 0) .
$$

This condition allows us to write the map in the form

$$
\Psi(\Theta, \mathcal{I})=(\Theta+F(\Theta, \mathcal{I}), G(\Theta, \mathcal{I}))
$$

where $F$ and $G$ are $2 \pi$-periodic and of class $C^{1}$. In particular $\Psi$ induces a map from $\mathbb{T}^{N} \times \mathcal{D}$ into $\mathbb{T}^{N} \times \mathbb{R}^{N}$

We say that $\Psi$ has the property of monotone twist if, for every $(\Theta, \mathcal{I}) \in \mathbb{R}^{N} \times \mathcal{D}$ and $\xi \in \mathbb{R}^{N} \backslash\{0\}$,

$$
\left\langle\partial_{\mathcal{I}} F(\Theta, \mathcal{I}) \xi, \xi\right\rangle>0
$$

Equivalently, the symmetric matrix $\frac{1}{2}\left[\partial_{\mathcal{I}} F+\left(\partial_{\mathcal{I}} F\right)^{t}\right]$ is positive definite. 
To interpret this condition we first obtain an important inequality. Given $\Theta \in \mathbb{R}^{N}$ and $\mathcal{I}_{1}, \mathcal{I}_{2} \in \mathcal{D}$, the convexity of $\mathcal{D}$ leads to

$$
F\left(\Theta, \mathcal{I}_{1}\right)-F\left(\Theta, \mathcal{I}_{2}\right)=\left(\int_{0}^{1} \partial_{\mathcal{I}} F\left(\Theta, \mathcal{I}_{2}+s\left(\mathcal{I}_{1}-\mathcal{I}_{2}\right)\right) d s\right)\left(\mathcal{I}_{1}-\mathcal{I}_{2}\right)
$$

When the condition $(2 \cdot 2)$ holds we obtain

$$
\left\langle F\left(\Theta, \mathcal{I}_{1}\right)-F\left(\Theta, \mathcal{I}_{2}\right), \mathcal{I}_{1}-\mathcal{I}_{2}\right\rangle>0
$$

if $\mathcal{I}_{1} \neq \mathcal{I}_{2}$. This means that the map $\mathcal{I} \in \mathcal{D} \mapsto F(\Theta, \mathcal{I}) \in \mathbb{R}^{N}$ is monotone in the sense of Functional Analysis. Note that, in particular, $F(\Theta, \cdot)$ is one-to-one. A geometric property that will be important later can be deduced from here. Consider the family of sets

$$
\mathcal{R}_{\Theta_{0}}=\left\{(\Theta, \mathcal{I}) \in \mathbb{R}^{N} \times \mathcal{D}: \Theta=\Theta_{0}\right\}
$$

with $\Theta_{0} \in \mathbb{R}^{N}$. Then the image $\Psi\left(\mathcal{R}_{\Theta_{0}}\right)$ will intersect any set $\mathcal{R}_{\Theta_{1}}$ at most once.

The twist condition has other consequences. In particular it implies that the determinant $\operatorname{det}\left(\partial_{\mathcal{I}} F\right)$ is positive on $\mathbb{T}^{N} \times \mathcal{D}$. We can now apply the inverse function theorem to deduce that $F(\Theta, \cdot)$ is an orientation-preserving diffeomorphism mapping $\mathcal{D}$ onto the open set $F(\Theta, \mathcal{D}) \subset \mathbb{R}^{N}$.

In addition to the monotone twist condition we shall assume that $\Psi$ is exact symplectic. This means that there exists a $2 \pi$-periodic function $V: \mathbb{R}^{N} \times \mathcal{D} \rightarrow \mathbb{R}$ of class $C^{1}$ such that

$$
d V=\Psi^{*} \lambda-\lambda
$$

where $\lambda$ is the 1 -form $\lambda=\sum_{i=1}^{N} I_{i} d \theta_{i}$ and $\Psi^{*} \lambda$ is the pull-back of $\lambda$ through $\Psi$. More explicitly,

$$
\partial_{\Theta} V=G^{t}+G^{t}\left(\partial_{\Theta} F\right)-\mathcal{I}^{t}, \quad \partial_{\mathcal{I}} V=G^{t}\left(\partial_{\mathcal{I}} F\right) .
$$

We are ready to state our result, which is inspired by [10, Theorem 2.1].

THEOREM $2 \cdot 1$. Assume that $\Psi$ is a $C^{1}$ map satisfying the above conditions $(2 \cdot 1),(2 \cdot 2)$ and $(2 \cdot 3)$. In addition, suppose that there exists $\nu \in \mathbb{Z}^{N}$ such that

$$
2 \pi \nu \in F(\Theta, \mathcal{D})
$$

for every $\Theta \in \mathbb{R}^{N}$. Then the equation

$$
\Psi(\Theta, \mathcal{I})=(\Theta+2 \pi \nu, \mathcal{I})
$$

has at least $N+1$ solutions that are geometrically different. In particular the map induced on $\mathbb{T}^{N} \times \mathcal{D}$ has at least $N+1$ fixed points.

Remark $2 \cdot 2$. Some remarks are now in order.

(i) When $N=1$ the set $\mathcal{D}$ is an open interval, say $\mathcal{D}=] a, b[$ with $-\infty \leqslant a<b \leqslant$ $+\infty$. The function $F(\Theta, \cdot)$ is strictly increasing and so it admits an extension mapping $[a, b]$ onto $[A, B]$ with $-\infty \leqslant A<B \leqslant+\infty$. The condition (2.5) can be reformulated as

$$
F(\Theta, a)<2 \pi \nu<F(\Theta, b)
$$

for each $\Theta \in \mathbb{R}$. It is customary to say that $\Psi$ has a twist on the boundary when $(2 \cdot 6)$ holds. This condition is unrelated to $(2 \cdot 2)$ and so the term twist is employed in two different senses. 
(ii) For higher dimension the condition (2.5) can be expressed in terms of Brouwer degree. Namely, for each $\Theta \in \mathbb{R}^{N}$

$$
\mathrm{d}\left(F(\Theta, \cdot), \Omega_{\Theta}, 2 \pi \nu\right)=1
$$

where $\Omega_{\Theta}$ is some open and bounded subset of $\mathbb{R}^{N}$ whose closure $\bar{\Omega}_{\Theta}$ is contained in $\mathcal{D}$. To justify this statement we recall some properties of the topological degree. Given a homeomorphism $h: \Omega \rightarrow \Omega_{1}$ between two open and bounded subsets of $\mathbb{R}^{N}, \mathrm{~d}(h, \Omega, p)= \pm 1$ if $p \in \Omega_{1}$. This is a consequence of the multiplication theorem applied to the composition $h^{-1} \circ h=\mathrm{id}_{\Omega}$. The positive sign corresponds to the orientation-preserving case. In our situation the map $\mathcal{I} \mapsto F(\Theta, \mathcal{I})$ is an orientation-preserving homeomorphism from $\mathcal{D}$ onto $F(\Theta, \mathcal{D})$. We can restrict it to some bounded and open sets $\Omega_{\Theta}$ and $\widehat{\Omega}_{\Theta}$ with $p=2 \pi \nu \in \widehat{\Omega}_{\Theta}$.

(iii) A different extension of (2.6) to higher dimension was introduced in [10]. Assuming that $\mathcal{D}$ is bounded and $\Psi$ has a continuous extension to $\mathbb{R}^{N} \times \overline{\mathcal{D}}$, it was assumed that

$$
\left\langle F(\Theta, \mathcal{I}), \mathcal{I}-\mathcal{I}_{*}\right\rangle>0
$$

for each $\Theta \in \mathbb{R}^{N}$ and $\mathcal{I} \in \partial \mathcal{D}$. Here $\partial \mathcal{D}$ is the boundary of $\mathcal{D}$ and $\mathcal{I}_{*}$ is a fixed point lying in $\mathcal{D}$. Under this assumption it is easy to check that $F(\Theta, \cdot)$ is linearly homotopic to the map $\mathcal{I} \mapsto \mathcal{I}-\mathcal{I}_{*}$ on the domain $\mathcal{D}$. This means that

$$
\lambda F(\Theta, \mathcal{I})+(1-\lambda)\left(\mathcal{I}-\mathcal{I}_{*}\right) \neq 0
$$

if $\lambda \in[0,1]$ and $\mathcal{I} \in \partial \mathcal{D}$. In consequence $\mathrm{d}(F(\Theta, \cdot), \mathcal{D}, 0)=1$ and the condition $(2 \cdot 5)$ holds with $\nu=0$.

(iv) The condition $(2 \cdot 2)$ could be replaced by

$$
\left\langle\partial_{\mathcal{I}} F(\Theta, \mathcal{I}) \xi, \xi\right\rangle<0,
$$

namely, the symmetric matrix $\frac{1}{2}\left[\partial_{\mathcal{I}} F+\left(\partial_{\mathcal{I}} F\right)^{t}\right]$ is negative definite. This corresponds to changing $\Theta$ with $-\Theta$ on the torus $\mathbb{T}^{N}$.

Proof. We must solve the system

$$
F(\Theta, \mathcal{I})=2 \pi \nu, \quad G(\Theta, \mathcal{I})=\mathcal{I} .
$$

If we freeze the angle variable $\Theta$ in the first equation, the previous discussions imply the existence of a unique action $\mathcal{I}=\mathcal{I}(\Theta)$ solving

$$
F(\Theta, \mathcal{I}(\Theta))=2 \pi \nu
$$

Here we have used the assumption $(2 \cdot 5)$. The implicit function theorem can be applied to deduce that $\Theta \mapsto \mathcal{I}(\Theta)$ is of class $C^{1}$ and

$$
\partial_{\Theta} \mathcal{I}=-\left(\partial_{\mathcal{I}} F\right)^{-1}\left(\partial_{\Theta} F\right) .
$$

The periodicity of $F$ together with the uniqueness of $\mathcal{I}(\Theta)$ imply that the map $\mathcal{I}=\mathcal{I}(\Theta)$ is $2 \pi$-periodic. After solving the first equation we must concentrate on the reduced system

$$
G(\Theta, \mathcal{I}(\Theta))=\mathcal{I}(\Theta) .
$$

The key to solve it is to observe that it has a variational structure. The function

$$
W(\Theta)=V(\Theta, \mathcal{I}(\Theta))
$$


is such that

$$
\nabla W(\Theta)=G(\Theta, \mathcal{I}(\Theta))-\mathcal{I}(\Theta) .
$$

To check the above identity we observe that $\partial_{\Theta} W=(\nabla W)^{t}$ and apply chain rule together with $(2 \cdot 4)$ and $(2 \cdot 7)$,

$$
\begin{aligned}
\partial_{\Theta} W & =\partial_{\Theta} V+\left(\partial_{\mathcal{I}} V\right)\left(\partial_{\Theta} \mathcal{I}\right) \\
& =G^{t}+G^{t}\left(\partial_{\Theta} F\right)-\mathcal{I}^{t}-G^{t}\left(\partial_{\mathcal{I}} F\right)\left(\partial_{\mathcal{I}} F\right)^{-1}\left(\partial_{\Theta} F\right)=(G-\mathcal{I})^{t} .
\end{aligned}
$$

The classical Lusternik-Schnirelmann theory [8] implies that the function on $\mathbb{T}^{N}$ induced by $W$ has at least $N+1$ critical points. Then there exist $\Theta_{1}, \ldots, \Theta_{N+1} \in \mathbb{R}^{N}$, geometrically different and such that $\nabla W\left(\Theta_{i}\right)=0, i=1, \ldots, N+1$. The points $\left(\Theta_{i}, \mathcal{I}\left(\Theta_{i}\right)\right)$ are the searched solutions of the system. There is a delicate point on the smoothness of $W$ (and hence of $\Psi$ ) that must be treated. The original approach by Lusternik and Schnirelmann dealt with functions of class $C^{2}$. This condition was imposed to guarantee the uniqueness for the Cauchy problem of the gradient system $\Theta^{\prime}=-\nabla W(\Theta)$. More modern approaches use the pseudo-gradient flow and only require class $C^{1}$ for $W$.

We now develop a consequence of Theorem $2 \cdot 1$ which deals with the case when the map $\Psi$ is the $k$-th iterate (for an integer $k \geqslant 2$ ) of an exact symplectic embedding $\Phi$; that is, $\Psi=\Phi^{k}$. In this case we can refine the conclusion about multiplicity. First we need to establish a framework. The map $\Phi: \mathbb{R}^{N} \times \mathcal{D} \rightarrow \mathbb{R}^{N} \times \mathbb{R}^{N}$ will be an exact symplectic embedding of class $C^{1}$ satisfying

$$
\Phi(\Theta+2 \pi \chi, \mathcal{I})=\Phi(\Theta, \mathcal{I})+(2 \pi \chi, 0) .
$$

The set $\mathcal{D}$ is open but we do not need to impose the convexity. Since $\Phi$ is not a self-map of $\mathbb{R}^{N} \times \mathcal{D}$, the iterates will be defined on smaller domains. Let us assume that $\mathcal{D}_{k}$ is an open and convex subset of $\mathcal{D}$ with the following property: given $(\Theta, \mathcal{I}) \in \mathbb{R}^{N} \times \mathcal{D}_{k}$, the sequence of iterates $\Phi^{h}(\Theta, \mathcal{I})$ is well defined for $h=1,2, \ldots, k$.

Note that some of the points of $\Phi^{h}(\Theta, \mathcal{I})$ can lie outside $\mathbb{R}^{N} \times \mathcal{D}_{k}$ but all the iterates are well defined on $\mathbb{R}^{N} \times \mathcal{D}_{k}$. This fact is crucial to prove that $\Phi^{k}=\Psi$ is an exact symplectic embedding on $\mathbb{R}^{N} \times \mathcal{D}_{k}$. Indeed, using standard properties of exterior differentiation,

$$
\begin{aligned}
d\left(V+V \circ \Phi+\cdots+V \circ \Phi^{k-1}\right) & =d V+\Phi^{*}(d V)+\cdots+\left(\Phi^{k-1}\right)^{*}(d V) \\
& =\Phi^{*} \lambda-\lambda+\left(\Phi^{2}\right)^{*} \lambda-\Phi^{*} \lambda+\cdots+\left(\Phi^{k}\right)^{*} \lambda-\left(\Phi^{k-1}\right)^{*} \lambda \\
& =\left(\Phi^{k}\right)^{*} \lambda-\lambda=\Psi^{*} \lambda-\lambda .
\end{aligned}
$$

In contrast, the monotone twist condition is not inherited by iterates. As an example assume $N=1$ and consider the standard map

$$
\Phi(\Theta, \mathcal{I})=(\Theta+\mathcal{I}, \mathcal{I}+\lambda \sin (\Theta+\mathcal{I})),
$$

where $\lambda$ is a real parameter. This map is exact symplectic and has monotone twist on the whole cylinder $\mathbb{T} \times \mathbb{R}$. In consequence also $\Psi=\Phi^{2}$ is exact symplectic but we will show that it does not have monotone twist when $|\lambda|$ is large. The second iterate $\left(\Theta_{2}, \mathcal{I}_{2}\right)=\Psi(\Theta, \mathcal{I})$ admits an explicit description with

$$
\Theta_{2}=\Theta+2 \mathcal{I}+\lambda \sin (\Theta+\mathcal{I})
$$

Then $\frac{\partial \Theta_{2}}{\partial \mathcal{I}}$ changes sign if $|\lambda|>2$. This fact will force us to impose the monotone condition on $\Psi$ and not on $\Phi$. 
Another difficulty will be concerned with the multiplicity of solutions of the equation

$$
\Psi(\Theta, \mathcal{I})=(\Theta+2 \pi \nu, \mathcal{I}), \quad(\Theta, \mathcal{I}) \in \mathbb{R}^{N} \times \mathcal{D}_{k} .
$$

Given a solution $\left(\Theta^{*}, \mathcal{I}^{*}\right)$, we can define the sequence of iterates $\Phi\left(\Theta^{*}, \mathcal{I}^{*}\right), \Phi^{2}\left(\Theta^{*}, \mathcal{I}^{*}\right), \ldots$, $\Phi^{k-1}\left(\Theta^{*}, \mathcal{I}^{*}\right)$. It might happen that some of these points do not belong to the domain of $\Psi$, but if any of these points belongs to $\mathbb{R}^{N} \times \mathcal{D}_{k}$, then it is also a solution of $(2 \cdot 8)$.

Finally, we mention that the notation

$$
\Psi(\Theta, \mathcal{I})=(\Theta+F(\Theta, \mathcal{I}), G(\Theta, \mathcal{I}))
$$

is still valid. Now $F$ and $G$ are $2 \pi$-periodic in $\mathbb{R}^{N} \times \mathcal{D}_{k}$.

Corollary 2.3. In the previous setting assume that $\Psi=\Phi^{k}$ satisfies the monotone twist condition on $\mathbb{R}^{N} \times \mathcal{D}_{k}$ and, for some $\nu \in \mathbb{Z}^{N}$ and each $\Theta \in \mathbb{R}^{N}$,

$$
2 \pi \nu \in F\left(\Theta, \mathcal{D}_{k}\right) .
$$

Then the equation $(2 \cdot 8)$ has at least $N+1$ solutions $\left(\Theta_{i}, \mathcal{I}_{i}\right), i=1, \ldots, N+1$, that are geometrically distinct and produce different periodic $\Phi$-orbits.

Note that the minimal period of $\Pi\left(\Theta_{i}, \mathcal{I}_{i}\right)$ with respect to $\Phi$ is not necessarily $k$. It can be any divisor of $k$.

Proof. We replace $\mathcal{D}$ by $\mathcal{D}_{k}$ and repeat the first part of the proof of the Theorem. In this way we find a $2 \pi$-periodic $C^{1}$ map $\mathcal{I}=\mathcal{I}(\Theta)$ solving

$$
F(\Theta, \mathcal{I}(\Theta))=2 \pi \nu
$$

Moreover (2.8) becomes equivalent to

$$
\nabla W=0,
$$

with

$$
W(\Theta)=\sum_{h=0}^{k-1} V\left(\Phi^{h}(\Theta, \mathcal{I}(\Theta))\right) .
$$

Here $V$ is the function satisfying $d V=\Phi^{*} \lambda-\lambda$. As already observed in the original work by Lusternik and Schnirelmann, there are two possible situations for a function on the torus: either there exists infinitely many critical points or there exist $N+1$ critical points $\Theta_{1}, \ldots, \Theta_{N+1}$ that lie on different critical levels, say

$$
W\left(\Theta_{1}\right)>W\left(\Theta_{2}\right)>\cdots>W\left(\Theta_{N+1}\right) .
$$

In the first situation the equation (2.8) has infinitely many solutions that are geometrically different. This implies that the conclusion of the Corollary holds. From now on we assume that we are in the second situation and (2.9) holds. Define $\mathcal{I}_{i}=\mathcal{I}\left(\Theta_{i}\right)$ so that $\left(\Theta_{i}, \mathcal{I}_{i}\right)$ is a solution of $(2 \cdot 8)$. From previous discussions we know that the points $\Phi^{r}\left(\Theta_{i}, \mathcal{I}_{i}\right)=\left(\Theta_{i}^{(r)}, \mathcal{I}_{i}^{(r)}\right)$ are also solutions if $\mathcal{I}_{i}^{(r)} \in \mathcal{D}_{k}$. In such a case the definition of the map $\mathcal{I}(\Theta)$ implies that $\mathcal{I}\left(\Theta_{i}^{(r)}\right)=\mathcal{I}_{i}^{(r)}$. Then

$$
W\left(\Theta_{i}^{(r)}\right)=\sum_{h=0}^{k-1} V\left(\Phi^{h}\left(\Theta_{i}^{(r)}, \mathcal{I}_{i}^{(r)}\right)\right)=\sum_{h=0}^{k-1} V\left(\Phi^{r+h}\left(\Theta_{i}, \mathcal{I}_{i}\right)\right)=W\left(\Theta_{i}\right) .
$$

In other words, the points on the $\Phi$-orbit of $\left(\Theta_{i}, \mathcal{I}_{i}\right)$ lie on the same critical level as soon 
as they belong to $\mathbb{R}^{N} \times \mathcal{D}_{k}$. This shows that the periodic orbits produced by the points $\left(\Theta_{1}, \mathcal{I}_{1}\right), \ldots,\left(\Theta_{N+1}, \mathcal{I}_{N+1}\right)$ are different.

For the applications we have in mind the maps $\Phi$ and $\Psi$ will be time maps associated to a Hamiltonian system of the type

$$
\left\{\begin{array}{l}
\Theta^{\prime}=\partial_{\mathcal{I}} H(t, \Theta, \mathcal{I}) \\
\mathcal{I}^{\prime}=-\partial_{\Theta} H(t, \Theta, \mathcal{I})
\end{array}\right.
$$

where $H: \mathbb{R} \times \mathbb{R}^{N} \times \mathcal{E} \rightarrow \mathbb{R}$ is a continuous function, $2 \pi$-periodic in the variable $\Theta$, such that the all the derivatives up to the second order with respect to $\Theta$ and $\mathcal{I}$ exist and are continuous on $\mathbb{R} \times \mathbb{R}^{N} \times \mathcal{E}$. The set $\mathcal{E}$ is an open subset of $\mathbb{R}^{N}$ and we also assume that there exists an open and convex set $\mathcal{D} \subset \mathcal{E}$ such that if $\left(\Theta_{0}, \mathcal{I}_{0}\right) \in \mathcal{D}$, the solution of $(2 \cdot 10)$ satisfying the initial condition $(\Theta(0), \mathcal{I}(0))=\left(\Theta_{0}, \mathcal{I}_{0}\right)$ is well defined on $[0, \tau]$ for some fixed $\tau>0$. Let $\left(\Theta\left(t ; \Theta_{0}, \mathcal{I}_{0}\right), \mathcal{I}\left(t ; \Theta_{0}, \mathcal{I}_{0}\right)\right)$ be this solutions and consider the $\tau$-map

$$
\left(\Theta_{0}, \mathcal{I}_{0}\right) \in \mathbb{R}^{N} \times \mathcal{D} \mapsto \Psi_{\tau}\left(\Theta_{0}, \mathcal{I}_{0}\right)=\left(\Theta\left(\tau ; \Theta_{0}, \mathcal{I}_{0}\right), \mathcal{I}\left(\tau ; \Theta_{0}, \mathcal{I}_{0}\right)\right) .
$$

We will prove that this map satisfies the conditions of the previous results, except perhaps for the monotone twist condition.

Proposition 2.4. The map $\Psi_{\tau}$ is an exact symplectic embedding of class $C^{1}$ satisfying $(2 \cdot 1)$.

Sketch of the proof. The only "not well known" part of the statement consists in the fact that $\Psi_{\tau}$ is exact symplectic (notice, however, that no smoothness in the time variable is required to ensure the smoothness of $\Psi_{\tau}$, see [7]). A possible way to prove it can be the following. At first, write the form $\Psi_{\tau}^{*} \lambda-\lambda$ as

$$
\Psi_{\tau}^{*} \lambda-\lambda=\sum_{i=1}^{N}\left(A_{i}(\Theta, \mathcal{I}) d \theta_{i}+B_{i}(\Theta, \mathcal{I}) d I_{i}\right)
$$

by definition, $\Psi_{\tau}$ is exact symplectic if and only if such a form has a primitive $2 \pi$-periodic in $\Theta$. By direct construction, we can see that this is the case if and only if, for every $(\Theta, \mathcal{I}) \in \mathbb{R}^{N} \times \mathcal{D}$, it holds that

$$
\int_{0}^{2 \pi} A_{i}\left(\theta_{1}, \ldots, \theta_{i-1}, s, \theta_{i+1}, \ldots, \theta_{N}, \mathcal{I}\right) d s=0, \quad i=1, \ldots, N,
$$

and this in turn corresponds to $\int_{\gamma}\left(\Psi_{\tau}^{*} \lambda-\lambda\right)=0$, being $\gamma$ the path

$$
[0,2 \pi] \ni s \mapsto \gamma(s)=\left(\theta_{1}, \ldots, \theta_{i-1}, s, \theta_{i+1}, \ldots, \theta_{N}, \mathcal{I}\right) .
$$

To see this, we can use the trick described in [1] , based on the Poincaré-Cartan differential form $\Lambda=\lambda-H d t$. First, one constructs a surface $S$ parameterized by

$$
(s, \sigma) \ni[0,2 \pi] \times[0, \tau] \mapsto\left(\Psi_{\sigma}(\gamma(s)), \sigma\right) \in \mathbb{R}^{2 N+1} .
$$

Since $d \Lambda=0$ on $S$, Stokes' theorem implies that $\int_{\partial S} \Lambda=0$. In the evaluation of this integral, contributions for $\sigma \in] 0, \tau[$ vanish in view of the $2 \pi$-periodicity in $\Theta$ of $H$, so that we actually have $\int_{\partial S_{0}} \Lambda=\int_{\partial S_{\tau}} \Lambda$, being $\partial S_{0}, \partial S_{\tau}$ the sections of $\partial S$ for $\sigma=$ $0, \tau$ respectively. But now, $\partial S_{0}$ is parameterized by $\gamma(s)$ and $\partial S_{\tau}$ by $\Psi_{\tau}(\gamma(s))$. Since, moreover, $\Lambda=\lambda$ on both sections, we finally find $\int_{\gamma} \lambda=\int_{\Psi_{\tau}(\gamma(s))} \lambda$, which was our claim. 
Remark 2.5. It is worth noticing that, if the $2 \pi$-periodicity in $\Theta$ of the Hamiltonian function $H$ is replaced by the $2 \pi$-periodicity in $\Theta$ of the Hamiltonian vector field $\left(\nabla_{\mathcal{I}} H,-\nabla_{\Theta} H\right)$, the map $(2 \cdot 11)$ is still a $C^{1}$ symplectic embedding satisfying $(2 \cdot 1)$, but in general it is no more exact symplectic. This already happens for $N=1$. For instance, it is well known that the $\tau$-map associated with the planar differential system

$$
\Theta^{\prime}=\mathcal{I}, \quad \mathcal{I}^{\prime}=-\sin \Theta+f(t)
$$

is exact symplectic if and only if $\int_{0}^{\tau} f(t) d t=0$ [6]. Indeed, the vector field in $(2 \cdot 12)$ is Hamiltonian and $2 \pi$-periodic in $\Theta$, but its Hamiltonian function is not. Notice that, if $\int_{0}^{\tau} f(t) d t=0$, the $\tau$-map associated with $(2 \cdot 12)$ coincides with the $\tau$-map of the differential system

$$
\Theta^{\prime}=\mathcal{I}+\int_{0}^{t} f(s) d s, \quad \mathcal{I}^{\prime}=-\sin \Theta
$$

which is indeed exact symplectic, since $(2 \cdot 13)$ comes from the Hamiltonian $H(t, \Theta, \mathcal{I})=$ $\frac{1}{2} \mathcal{I}^{2}+\left(\int_{0}^{t} f(s) d s\right) \mathcal{I}-\cos \Theta, 2 \pi$-periodic in $\Theta$.

\section{Periodic solutions of weakly coupled superlinear systems}

We will work on the plane $\mathbb{R}^{2}$ with points $x=\left(x_{1}, x_{2}\right)$. The euclidean norm will be denoted by $|x|$. Given a $2 \times 2$ matrix $A$, the associated matrix norm will be $|A|$.

Let $P=P(t, x), P: \mathbb{R} \times \mathbb{R}^{2} \rightarrow \mathbb{R}$, be a function in $C^{0,2}\left(\mathbb{R} /(T \mathbb{Z}) \times \mathbb{R}^{2}\right)$. This means that $P$ is continuous, $T$-periodic in $t$ and the partial derivatives $\partial_{x_{i}} P=\partial_{i} P, \partial_{x_{i} x_{j}}^{2} P=\partial_{i j} P$ exist and are continuous in $(t, x) \in \mathbb{R} \times \mathbb{R}^{2}$. Sometimes we will employ the notations

$$
\nabla P=\left(\partial_{1} P, \partial_{2} P\right)^{t}, \quad D^{2} P=\left(\begin{array}{ll}
\partial_{11} P & \partial_{12} P \\
\partial_{12} P & \partial_{22} P
\end{array}\right) .
$$

We assume that there exists a constant $C>0$ such that

$$
|\nabla P(t, x)|+\left|D^{2} P(t, x)\right| \leqslant C \quad \text { for every }(t, x) \in \mathbb{R} \times \mathbb{R}^{2} .
$$

In addition we impose that the second derivative $D^{2} P$ vanishes at infinity in a suitable sense. More precisely, for each $\epsilon \in] 0,1[$ we define the set

$$
K_{\epsilon}=\left\{x \in \mathbb{R}^{2}: \epsilon\left|x_{1}\right| \leqslant\left|x_{2}\right| \leqslant \frac{1}{\epsilon}\left|x_{1}\right|\right\}
$$

and assume that for each $\epsilon \in] 0,1[$

$$
\lim _{\substack{|x| \rightarrow \infty \\ x \in K_{\epsilon}}}\left|D^{2} P(t, x)\right|=0 \quad \text { uniformly in } t \in \mathbb{R} .
$$

Potentials of the type

$$
P\left(t, x_{1}, x_{2}\right)=\varphi_{1}\left(x_{1}\right) \varphi_{2}\left(x_{2}\right)+p_{1}(t) x_{1}+p_{2}(t) x_{2}
$$

satisfy the above assumptions if the functions $\varphi_{1}$ and $\varphi_{2}$ are bounded, of class $C^{2}$, with

$$
\varphi_{i}^{\prime}(x) \rightarrow 0, \quad \varphi_{i}^{\prime \prime}(x) \rightarrow 0, \quad \text { as } \quad|x| \rightarrow \infty, \quad i=1,2,
$$

and $p_{i}(t)$ are continuous and $T$-periodic. The example presented in the introduction belongs to this family. The condition $(3 \cdot 2)$ cannot be satisfied by a potential of the type

$$
P\left(t, x_{1}, x_{2}\right)=\varphi\left(x_{1}-x_{2}\right)+p_{1}(t) x_{1}+p_{2}(t) x_{2} .
$$


Due to its mechanical significance this class deserves further study.

We are interested in the existence of periodic solutions with period $k T, k=1,2, \ldots$, of the system

$$
x_{i}^{\prime \prime}+x_{i}^{3}=\partial_{i} P(t, x), \quad i=1,2 .
$$

Let us recall that these solutions are called subharmonic solutions of order $k \geqslant 2$ if they do not admit a period $l T$ with $l=1, \ldots, k-1$. Given a subharmonic solution $x(t)$, the time translations $x(t+T), x(t+2 T), \ldots, x(t+(k-1) T)$ are also subharmonic solutions. This family will be called a periodicity class. The following notion will be useful to find subharmonic solutions. A point $\nu=\left(\nu_{1}, \ldots, \nu_{d}\right) \in \mathbb{N}^{d}$ is visible from the origin if the segment in $\mathbb{R}^{d}$ joining the origin with the point $\nu$ does not contain any other point in $\mathbb{N}^{d}$.

TheOREm 3.1. Assume that the conditions (3.1) and $(3 \cdot 2)$ hold and fix an integer $k \geqslant 1$ and numbers $\alpha, \beta \in \mathbb{R}$ with $0<\alpha<\beta$. Then there exists $\nu^{*}=\nu^{*}(k, \alpha, \beta)>0$ such that, for every $\nu=\left(\nu_{1}, \nu_{2}\right) \in \mathbb{N}^{2}$ with

$$
\alpha \leqslant \frac{\nu_{2}}{\nu_{1}} \leqslant \beta \quad \text { and } \quad \nu_{1}+\nu_{2} \geqslant \nu^{*}
$$

the system $(3 \cdot 3)$ has at least three periodic solutions $\left(x_{1}(t), x_{2}(t)\right)$ of period $k T$ and such that $x_{i}(t)$ has exactly $\nu_{i}$ zeros on $\left[0, k T\left[\right.\right.$ for $i=1,2$. Moreover, if $k \geqslant 2$ and $\left(k, \nu_{1}, \nu_{2}\right) \in$ $\mathbb{N}^{3}$ is visible from the origin, these solutions are subharmonic solutions of order $k$ lying in different periodicity classes.

As an illustration we can think in the visibility condition for $k=2$. In this case it means that at least one of the two numbers $\nu_{1}, \nu_{2}$ is odd.

The proof of this theorem will be divided in several steps. Our first task will be to transform the system $(3 \cdot 3)$ into another system adapted to the abstract setting of Section 2 .

\subsection{A change of variable}

For $P=0$ the system $(3 \cdot 3)$ is Liouville integrable and the associated action-angle variables are defined via the map

$$
\left.\varphi:(\Theta, \mathcal{I}) \in \mathbb{T}^{2} \times\right] 0, \infty\left[{ }^{2} \mapsto\left(x_{1}, y_{1}, x_{2}, y_{2}\right) \in\left(\mathbb{R}^{2} \backslash\{0\}\right) \times\left(\mathbb{R}^{2} \backslash\{0\}\right)\right.
$$

with $\Theta=\left(\theta_{1}, \theta_{2}\right), \mathcal{I}=\left(I_{1}, I_{2}\right)$ and

$$
\left\{\begin{array}{l}
x_{i}=\gamma I_{i}^{1 / 3} c\left(\theta_{i}\right) \\
y_{i}=\gamma^{2} I_{i}^{2 / 3} s\left(\theta_{i}\right) .
\end{array}\right.
$$

The function $c(t)$ is the unique solution of the Duffing equation $x^{\prime \prime}+x^{3}=0$ with minimal period $2 \pi$ and $c(0)>0, c^{\prime}(0)=0$. The function $s(t)$ is defined as the derivative, $s(t)=$ $c^{\prime}(t)$. Finally the number $\gamma>0$ is adjusted so that

$$
d x_{i} \wedge d y_{i}=d I_{i} \wedge d \theta_{i} .
$$

Now it is easy to verify that $\varphi$ is a symplectic diffeomorphism and so the Hamiltonian system associated to $(3 \cdot 3)$

$$
\begin{gathered}
x^{\prime}=\partial_{y} H, \quad y^{\prime}=-\partial_{x} H \\
H(t, x, y)=\frac{1}{2}\left(y_{1}^{2}+y_{2}^{2}\right)+\frac{1}{4}\left(x_{1}^{4}+x_{2}^{4}\right)-P\left(t, x_{1}, x_{2}\right)
\end{gathered}
$$


is transformed into

$$
\begin{gathered}
\Theta^{\prime}=-\partial_{\mathcal{I}} \mathcal{H}(t, \Theta, \mathcal{I}), \quad \mathcal{I}^{\prime}=\partial_{\Theta} \mathcal{H}(t, \Theta, \mathcal{I}), \\
\mathcal{H}(t, \Theta, \mathcal{I})=\frac{3}{4} \omega\left(I_{1}^{4 / 3}+I_{2}^{4 / 3}\right)-P\left(t, \gamma I_{1}^{1 / 3} c\left(\theta_{1}\right), \gamma I_{2}^{1 / 3} c\left(\theta_{2}\right)\right) .
\end{gathered}
$$

The number $\omega>0$ is a constant depending on the transformation $\varphi$ which does not need to be specified.

The same change of variables was employed in $[4]$ to prove the existence of invariant tori.

Note that $\varphi$ does not cover the planes $x_{1}=y_{1}=0$ and $x_{2}=y_{2}=0$. The equivalence between the two systems must be understood on the phase spaces $\left.\mathbb{T}^{2} \times\right] 0, \infty\left[{ }^{2}\right.$ and $\left(\mathbb{R}^{2} \backslash\right.$ $\{0\}) \times\left(\mathbb{R}^{2} \backslash\{0\}\right)$. In particular the solutions of (3.5) with initial conditions $x_{1}\left(t_{0}\right)=$ $x_{1}^{\prime}\left(t_{0}\right)=0$ will lead to solutions of $(3 \cdot 6)$ having a maximal interval of existence $J \neq \mathbb{R}$. The same can be said for the conditions $x_{2}\left(t_{0}\right)=x_{2}^{\prime}\left(t_{0}\right)=0$. The periodic solutions in Theorem $3 \cdot 1$ will be produced via the system $(3 \cdot 6)$ and so they cannot satisfy any of these conditions.

Let us denote by $\left(\Theta\left(t ; \Theta_{0}, \mathcal{I}_{0}\right), \mathcal{I}\left(t ; \Theta_{0}, \mathcal{I}_{0}\right)\right)=(\Theta(t), \mathcal{I}(t))$ the unique solution of $(3 \cdot 6)$ satisfying $\Theta(0)=\Theta_{0}$ and $\mathcal{I}(0)=\mathcal{I}_{0}$. The coordinates will be denoted by $\theta_{i}\left(t ; \Theta_{0}, \mathcal{I}_{0}\right)=$ $\theta_{i}(t), I_{i}\left(t ; \Theta_{0}, \mathcal{I}_{0}\right)=I_{i}(t)$ and the initial conditions by $\Theta_{0}=\left(\theta_{10}, \theta_{20}\right), \mathcal{I}_{0}=\left(I_{10}, I_{20}\right)$.

\subsection{Estimates on actions and angles}

From now on we fix an integer $k \geqslant 1$ and assume $T=2 \pi$. We will prove that solutions with large initial actions are well defined on $[0,2 k \pi]$.

Lemma 3.2. There exists $l_{k}>0$ such that, for every $\Theta_{0} \in \mathbb{R}^{2}$ and $\left.\mathcal{I}_{0} \in\right] l_{k},+\infty\left[{ }^{2}\right.$, the solution $\left(\Theta\left(t ; \Theta_{0}, \mathcal{I}_{0}\right), \mathcal{I}\left(t ; \Theta_{0}, \mathcal{I}_{0}\right)\right)$ exists on $[0,2 k \pi]$ and satisfies

$$
\frac{I_{i 0}}{4} \leqslant I_{i}\left(t ; \Theta_{0}, \mathcal{I}_{0}\right) \leqslant 4 I_{i 0} \quad \text { for every } t \in[0,2 k \pi], i=1,2 .
$$

Proof. Let $\tau \in] 0,2 k \pi]$ be the supremum of the instants such that $I_{i 0} / 4<I_{i}\left(t ; \Theta_{0}, \mathcal{I}_{0}\right)<$ $4 I_{i 0}$ for $t \in[0, \tau[$ and $i=1,2$. From the system (3.6),

$$
I_{i}^{\prime}=-\gamma \partial_{i} P\left(t, \gamma I_{1}^{1 / 3} c\left(\theta_{1}\right), \gamma I_{2}^{1 / 3} c\left(\theta_{2}\right)\right) I_{i}^{1 / 3} s\left(\theta_{i}\right) .
$$

From $(3 \cdot 1)$, we find a constant $C_{1}>0$ such that

$$
\left|\frac{d}{d t} I_{i}(t)^{2 / 3}\right| \leqslant C_{1} \quad \text { for every } t \in[0, \tau[.
$$

Hence, for $t \in\left[0, \tau\left[\right.\right.$ and $I_{i 0}$ large enough, we have

$$
\left(\frac{I_{i 0}}{2}\right)^{2 / 3} \leqslant I_{i 0}^{2 / 3}-2 k \pi C_{1} \leqslant I_{i}(t)^{2 / 3} \leqslant I_{i 0}^{2 / 3}+2 k \pi C_{1} \leqslant\left(2 I_{i 0}\right)^{2 / 3} .
$$

Therefore $\tau=2 k \pi$ and (3.7) holds true.

The next result provides an estimate for the angles of those solutions with large actions.

Lemma 3.3. There exist $\widehat{l}_{k}>0$ and $M_{k}>0$ such that, for every $\Theta_{0} \in \mathbb{R}^{2}$ and $\mathcal{I}_{0} \in$ $\widehat{l}_{k},+\infty\left[^{2}\right.$,

$$
\frac{\omega}{4} I_{i 0}^{1 / 3} \leqslant\left|\theta_{i}^{\prime}\left(t ; \Theta_{0}, \mathcal{I}_{0}\right)\right| \leqslant 4 \omega I_{i 0}^{1 / 3}
$$


and

$$
\left|\theta_{i}\left(t ; \Theta_{0}, \mathcal{I}_{0}\right)-\theta_{i 0}+\omega\left(I_{i 0}\right)^{1 / 3} t\right| \leqslant \frac{M_{k}}{I_{i 0}^{1 / 3}}
$$

for every $t \in[0,2 k \pi]$ and $i=1,2$.

Proof. From the system (3.6),

$$
\theta_{i}^{\prime}=-\omega I_{i}^{1 / 3}+\frac{1}{3} \gamma \partial_{i} P\left(t, \gamma I_{1}^{1 / 3} c\left(\theta_{1}\right), \gamma I_{2}^{1 / 3} c\left(\theta_{2}\right)\right) I_{i}^{-2 / 3} c\left(\theta_{i}\right) .
$$

Then Lemma 3.2 and $(3 \cdot 1)$ immediately implies the existence of $\widehat{l}_{k}>0$ such that the desired estimate for $\left|\theta_{i}^{\prime}\right|$ holds true.

On the other hand, integrating $(3 \cdot 8)$ over $[0, t]$ and using again Lemma $3 \cdot 2$ and $(3 \cdot 1)$, we obtain

$$
\theta_{i}(t)-\theta_{i 0}+\omega\left(I_{i 0}\right)^{1 / 3} t=\omega \int_{0}^{t}\left(I_{i 0}^{1 / 3}-I_{i}(s)^{1 / 3}\right) d s+R(t)
$$

with $I_{i 0}^{2 / 3}|R(t)|$ bounded. To estimate the integral we proceed as in the proof of Lemma $3 \cdot 2$ and use the mean value theorem to obtain

$$
\left|I_{i 0}^{2 / 3}-I_{i}(s)^{2 / 3}\right| \leqslant C_{1} 2 k \pi
$$

Hence, for every $s \in[0, t]$,

$$
\left|I_{i 0}^{1 / 3}-I_{i}(s)^{1 / 3}\right|=\left|\frac{I_{i 0}^{2 / 3}-I_{i}(s)^{2 / 3}}{I_{i 0}^{1 / 3}+I_{i}(s)^{1 / 3}}\right| \leqslant \frac{C_{1} 2 k \pi}{\left(1+\left(\frac{1}{4}\right)^{1 / 3}\right) I_{i 0}^{1 / 3}}
$$

and the conclusion follows.

For the remaining estimates we need to fix $\epsilon>0$ and work on the set of initial conditions

$$
\mathcal{C}_{\epsilon}=\left\{\mathcal{I}_{0}=\left(I_{10}, I_{20}\right): 0<\epsilon I_{10} \leqslant I_{20} \leqslant \frac{1}{\epsilon} I_{10}\right\} .
$$

All constants will depend on $k$ and $\epsilon$. It is interesting to notice that on the set $\mathcal{C}_{\epsilon}$ the norm of vectors can be estimated by any of the two components, that is

$$
\left|\mathcal{I}_{0}\right| \leqslant \sqrt{\left(\frac{1}{\epsilon^{2}}+1\right)}\left|I_{i 0}\right|, \quad i=1,2 .
$$

We will consider quantities $F=F\left(t ; \Theta_{0}, \mathcal{I}_{0}\right)$ or $F=F\left(\Theta_{0}, \mathcal{I}_{0}\right)$. Asymptotic notations of the type

$$
F\left(t ; \Theta_{0}, \mathcal{I}_{0}\right)=O\left(\left|\mathcal{I}_{0}\right|^{\sigma}\right) \quad \text { as }\left|\mathcal{I}_{0}\right| \rightarrow \infty, \mathcal{I}_{0} \in \mathcal{C}_{\epsilon}
$$

will be employed. They hold for actions lying on $\mathcal{C}_{\epsilon}$ and it is understood that they hold uniformly with respect to the other variables $t \in[0,2 k \pi]$ and $\Theta_{0} \in \mathbb{R}^{2}$. As an example we observe that the previous proof implies that

$$
\frac{I_{i}\left(t ; \Theta_{0}, \mathcal{I}_{0}\right)}{I_{i 0}}=1+O\left(\left|\mathcal{I}_{0}\right|^{-2 / 3}\right) \quad \text { as }\left|\mathcal{I}_{0}\right| \rightarrow \infty, \mathcal{I}_{0} \in \mathcal{C}_{\epsilon}
$$

This formula as well as (3.7) will be used many times. 


\section{3·3. The variational equation}

Let us consider the linear system

$$
\zeta^{\prime}=J D^{2} \mathcal{H}\left(t, \Theta\left(t ; \Theta_{0}, \mathcal{I}_{0}\right), \mathcal{I}\left(t ; \Theta_{0}, \mathcal{I}_{0}\right)\right) \zeta
$$

where $\zeta$ is a $4 \times 2$ matrix satisfying the initial conditions

$$
\zeta_{1}(0)=0, \quad \zeta_{2}(0)=\mathrm{Id} .
$$

Here $\zeta_{1}$ and $\zeta_{2}$ are $2 \times 2$ sub-matrices so that $\zeta=\left(\begin{array}{l}\zeta_{1} \\ \zeta_{2}\end{array}\right)$, Id is the $2 \times 2$ identity matrix and $J=\left(\begin{array}{cc}0 & -\mathrm{Id} \\ \mathrm{Id} & 0\end{array}\right)$.

The theorem on differentiability with respect to initial conditions implies that

$$
\zeta_{1}(t)=\partial_{\mathcal{I}_{0}} \Theta\left(t ; \Theta_{0}, \mathcal{I}_{0}\right), \quad \zeta_{2}(t)=\partial_{\mathcal{I}_{0}} \mathcal{I}\left(t ; \Theta_{0}, \mathcal{I}_{0}\right) .
$$

To simplify the discussion the matrix $J D^{2} \mathcal{H}$ will be decomposed in $2 \times 2$ blocks

$$
J D^{2} \mathcal{H}=\left(\begin{array}{ll}
A & B \\
\Gamma & \Delta
\end{array}\right)
$$

with

$$
A=-\Delta^{t}=-\partial_{\Theta \mathcal{I}} \mathcal{H}, \quad B=-\partial_{\mathcal{I} \mathcal{I}} \mathcal{H}, \quad \Gamma=\partial_{\Theta \Theta} \mathcal{H}
$$

The most delicate computations will be those involving $\Gamma$ and we present the complete computations for this sub-matrix. They are obtained after differentiating twice the Hamiltonian $\mathcal{H}$,

$$
\begin{aligned}
& \Gamma=\left(\begin{array}{ll}
\Gamma_{11} & \Gamma_{12} \\
\Gamma_{12} & \Gamma_{22}
\end{array}\right) \\
& \Gamma_{i i}=\gamma \partial_{i} P I_{i}^{1 / 3} c\left(\theta_{i}\right)^{3}-\gamma^{2} \partial_{i i} P I_{i}^{2 / 3} s\left(\theta_{i}\right)^{2}, \quad \Gamma_{12}=-\gamma^{2} \partial_{12} P I_{1}^{1 / 3} I_{2}^{1 / 3} s\left(\theta_{1}\right) s\left(\theta_{2}\right) .
\end{aligned}
$$

The matrix $\Gamma$ satisfies

$$
\Gamma=O\left(\left|\mathcal{I}_{0}\right|^{2 / 3}\right) \quad \text { as }\left|\mathcal{I}_{0}\right| \rightarrow \infty, \mathcal{I}_{0} \in \mathcal{C}_{\epsilon} .
$$

Similar computations lead to

$$
A=O\left(\left|\mathcal{I}_{0}\right|^{-1 / 3}\right), \quad B=-\frac{\omega}{3} E^{-1}+O\left(\left|\mathcal{I}_{0}\right|^{-4 / 3}\right)
$$

where

$$
E=\left(\begin{array}{cc}
I_{10}^{2 / 3} & 0 \\
0 & I_{20}^{2 / 3}
\end{array}\right)
$$

The block $\Gamma$ has the dominant growth and this fact motivates the change of variables

$$
\eta_{1}=E \zeta_{1}, \quad \eta_{2}=\zeta_{2}
$$

A similar trick was employed in the proof of Lemma 5.1 in [5].

The linear system (3.9) is transformed into

$$
\eta^{\prime}=\mathcal{A}\left(t ; \Theta_{0}, \mathcal{I}_{0}\right) \eta, \quad \eta_{1}(0)=0, \eta_{2}(0)=\mathrm{Id}
$$

with

$$
\mathcal{A}=\left(\begin{array}{cc}
E A E^{-1} & E B \\
\Gamma E^{-1} & \Delta
\end{array}\right)
$$


The previous asymptotic expansions imply that the sub-matrices $E A E^{-1}$ and $\Delta$ are of order $\left|\mathcal{I}_{0}\right|^{-1 / 3}$ while $E B=-\frac{\omega}{3} \operatorname{Id}+O\left(\left|\mathcal{I}_{0}\right|^{-2 / 3}\right)$ and $\Gamma E^{-1}=O(1)$. In particular the matrix $\mathcal{A}$ is uniformly bounded.

\subsection{The monotone twist condition}

Proposition 3.4. It holds

$$
E \partial_{\mathcal{I}_{0}} \Theta\left(t ; \Theta_{0}, \mathcal{I}_{0}\right)=-\frac{\omega}{3} \operatorname{Id} t+o(1) \quad \text { as }\left|\mathcal{I}_{0}\right| \rightarrow \infty, \mathcal{I}_{0} \in \mathcal{C}_{\epsilon} .
$$

At first sight this result looks rather technical but it is a crucial step in the proof. It implies that the matrix $\partial_{\mathcal{I}_{0}} \Theta\left(2 k \pi ; \Theta_{0}, \mathcal{I}_{0}\right)$ is definite negative if $\mathcal{I}_{0} \in \mathcal{C}_{\epsilon}$ and $\left|\mathcal{I}_{0}\right|$ is large enough. To prove this we fix a number $\mu$ with $0<\mu<\omega k \pi$ and select $l_{*}>0$ so that

$$
\frac{\partial \theta_{i}}{\partial I_{i 0}}\left(2 k \pi ; \Theta_{0}, \mathcal{I}_{0}\right) \leqslant-\frac{\omega}{3} k \pi I_{i 0}^{-2 / 3} \quad \text { if } i=1,2
$$

and

$$
\left|\frac{\partial \theta_{i}}{\partial I_{j 0}}\left(2 k \pi ; \Theta_{0}, \mathcal{I}_{0}\right)\right| \leqslant \frac{\mu}{3} I_{j 0}^{-2 / 3} \quad \text { if } i \neq j .
$$

We are assuming $\mathcal{I}_{0} \in \mathcal{C}_{\epsilon}$ and $\left|\mathcal{I}_{0}\right| \geqslant l_{*}$. Then

$$
\operatorname{det}\left[\partial_{\mathcal{I}_{0}} \Theta\left(2 k \pi ; \Theta_{0}, \mathcal{I}_{0}\right)\right] \geqslant \frac{(\omega k \pi)^{2}-\mu^{2}}{9} I_{10}^{-2 / 3} I_{20}^{-2 / 3}>0 .
$$

To prove the proposition we need two preliminary results. The first result is concerned with the theory of functions. Given a compact interval $[a, b]$, the class of functions $f \in$ $C^{1}[a, b]$ satisfying $f^{\prime}(t) \neq 0$ for each $t \in[a, b]$ will be denoted by $\mathcal{E}^{1}[a, b]$. Also, we employ the notation

$$
m(f)=\min _{[a, b]}\left|f^{\prime}\right|, \quad M(f)=\max _{[a, b]}\left|f^{\prime}\right|
$$

for each $f \in \mathcal{E}^{1}[a, b]$.

Lemma 3.5. There exists a number $\sigma>0$, depending only on the function $c(\theta)$, such that

$$
\text { meas }\{t \in[a, b]:|c(f(t))|<\rho\} \leqslant \frac{\sigma}{m(f)}\left[\frac{M(f)}{2 \pi}(b-a)+2\right] \rho
$$

if $f \in \mathcal{E}^{1}[a, b]$ and $\rho>0$.

Here meas refers to the Lebesgue measure on the real line.

Proof. It is sufficient to prove the result when $\rho$ is small. Let $\theta_{*}<\theta^{*}$ be the two zeros of $c(\theta)$ lying in $[0,2 \pi]$. The function $c(\theta)$ is $2 \pi$-periodic and for small $\rho$ the set

$$
c_{\rho}=\{\theta \in \mathbb{R}:|c(\theta)|<\rho\}
$$

can be expressed as a disjoint union of open intervals $I_{n}$ and $J_{n}$ satisfying

$$
\begin{array}{cc}
c_{\rho}=\bigcup_{n \in \mathbb{Z}}\left(I_{n} \cup J_{n}\right), & \theta_{*} \in I_{0}, \theta^{*} \in J_{0}, \\
I_{n}=I_{0}+2 n \pi, & J_{n}=J_{0}+2 n \pi,
\end{array}
$$

and

$$
\text { meas }\left(I_{n}\right) \leqslant \sigma_{1} \rho, \quad \text { meas }\left(J_{n}\right) \leqslant \sigma_{2} \rho \text {. }
$$


Note that this is possible because $\theta_{*}$ and $\theta^{*}$ are simple zeros, $c\left(\theta_{*}\right)=c\left(\theta^{*}\right)=0, c^{\prime}\left(\theta_{*}\right)=$ $-c^{\prime}\left(\theta^{*}\right) \neq 0$.

Consider the set of integers

$$
\Lambda=\left\{n \in \mathbb{Z}:\left(I_{n} \cup J_{n}\right) \cap f([a, b]) \neq \emptyset\right\}
$$

and let $N=\# \Lambda$ be its cardinality. Then

$$
2 \pi(N-2) \leqslant|f(b)-f(a)| \leqslant M(f)(b-a),
$$

leading to the estimate for $N$,

$$
N \leqslant \frac{M(f)}{2 \pi}(b-a)+2
$$

The set $f^{-1}\left(I_{n}\right)$ is an open interval satisfying

$$
\text { meas } f^{-1}\left(I_{n}\right) \leqslant \frac{1}{m(f)} \text { meas }\left(I_{n}\right) \leqslant \frac{\sigma_{1}}{m(f)} \rho .
$$

A similar inequality holds for $f^{-1}\left(J_{n}\right)$. Then

$$
\text { meas }\{t \in[a, b]:|c(f(t))|<\rho\} \leqslant \operatorname{meas} \bigcup_{n \in \Lambda}\left(f^{-1}\left(I_{n}\right) \cup f^{-1}\left(I_{n}\right)\right) \leqslant N \frac{\sigma_{1}+\sigma_{2}}{m(f)} \rho .
$$

This estimate together with $(3 \cdot 10)$ leads to the conclusion.

The second preliminary result is concerned with the limit of the matrix $\mathcal{A}\left(\cdot ; \Theta_{0}, \mathcal{I}_{0}\right)$ in the $L^{1}$-topology.

Lemma 3·6. Let $\mathcal{A}_{\infty}$ be the constant $4 \times 4$ matrix $\left(\begin{array}{l}0-\frac{\omega}{3} \mathrm{Id} \\ 0 \\ 0\end{array}\right)$. Then

$$
\int_{0}^{2 k \pi}\left|\mathcal{A}\left(t ; \Theta_{0}, \mathcal{I}_{0}\right)-\mathcal{A}_{\infty}\right| d t=o(1) \quad \text { as }\left|\mathcal{I}_{0}\right| \rightarrow \infty, \mathcal{I}_{0} \in \mathcal{C}_{\epsilon} .
$$

Proof. The limits

$$
\int_{0}^{2 k \pi}\left|E A E^{-1}\right| d t \rightarrow 0, \quad \int_{0}^{2 k \pi}\left|E B+\frac{\omega}{3} \mathrm{Id}\right| d t \rightarrow 0, \quad \int_{0}^{2 k \pi}|\Delta| d t \rightarrow 0
$$

are a direct consequence of the asymptotic expansions for these sub-matrices. Moreover, the convergence is uniform in $\Theta_{0} \in \mathbb{R}^{2}$. The limit

$$
\int_{0}^{2 k \pi}\left|\Gamma E^{-1}\right| d t \rightarrow 0
$$

requires a more careful analysis. The explicit formulas for the coefficients $\Gamma_{i j}$ reveal that it is enough to prove that

$$
\int_{0}^{2 k \pi} Q\left(t ; \Theta_{0}, \mathcal{I}_{0}\right) d t \rightarrow 0 \quad \text { as }\left|\mathcal{I}_{0}\right| \rightarrow \infty, \mathcal{I}_{0} \in \mathcal{C}_{\epsilon}
$$

where

$$
Q\left(t ; \Theta_{0}, \mathcal{I}_{0}\right)=\left|D^{2} P(t, x(t))\right| \quad \text { and } \quad x(t)=\gamma\left(I_{1}(t)^{1 / 3} c\left(\theta_{1}(t)\right), I_{2}(t)^{1 / 3} c\left(\theta_{2}(t)\right)\right) .
$$

Let us proceed by contradiction and assume the existence of a number $\delta>0$ and sequences $\mathcal{I}_{0}^{(n)} \in \mathcal{C}_{\epsilon},\left|\mathcal{I}_{0}^{(n)}\right| \rightarrow \infty, \Theta_{0}^{(n)} \in \mathbb{R}^{2}$ such that

$$
\int_{0}^{2 k \pi} Q\left(t ; \Theta_{0}^{(n)}, \mathcal{I}_{0}^{(n)}\right) d t \geqslant \delta .
$$


We fix a number $\rho>0$ such that, for large $n$,

$$
8 \sigma\left(4 k+\frac{1}{\omega\left(I_{10}^{(n)}\right)^{1 / 3}}+\frac{1}{\omega\left(I_{20}^{(n)}\right)^{1 / 3}}\right) C \rho \leqslant \frac{\delta}{2} .
$$

Here $\sigma$ is the constant appearing in Lemma 3.5 and $C$ is the bound for $Q$ coming from $(3 \cdot 1)$.

We know, by Lemma $3 \cdot 3$, that the functions $\theta_{i}^{(n)}=\theta_{i}\left(\cdot ; \Theta_{0}^{(n)}, \mathcal{I}_{0}^{(n)}\right)$ belong to $\mathcal{E}^{1}[0,2 k \pi]$ and

$$
m\left(\theta_{i}^{(n)}\right) \geqslant \frac{\omega}{4}\left(I_{i 0}^{(n)}\right)^{1 / 3}, \quad M\left(\theta_{i}^{(n)}\right) \leqslant 4 \omega\left(I_{i 0}^{(n)}\right)^{1 / 3}
$$

The previous lemma implies that the set

$$
A_{\rho, n}=\bigcup_{i=1}^{2}\left\{t \in[0,2 k \pi]:\left|c\left(\theta_{i}^{(n)}(t)\right)\right|<\rho\right\}
$$

satisfies

$$
\operatorname{meas}\left(A_{\rho, n}\right) \leqslant 8 \sigma\left(4 k+\frac{1}{\omega\left(I_{10}^{(n)}\right)^{1 / 3}}+\frac{1}{\omega\left(I_{20}^{(n)}\right)^{1 / 3}}\right) \rho .
$$

In consequence

$$
\int_{A_{\rho, n}} Q\left(t ; \Theta_{0}^{(n)}, \mathcal{I}_{0}^{(n)}\right) d t \leqslant \frac{\delta}{2}
$$

for large $n$.

To study the integral on the complement $[0,2 k \pi] \backslash A_{\rho, n}$ we first observe that there exists $\epsilon^{*}>0$, depending only on $\epsilon$ and $\rho$, such that if $n$ is large enough and $t \in[0,2 k \pi] \backslash A_{\rho, n}$ then

$$
x_{n}(t) \in \mathcal{K}_{\epsilon^{*}} .
$$

Here $x_{n}=\gamma\left(\left(I_{1}^{(n)}\right)^{1 / 3} c\left(\theta_{1}^{(n)}\right),\left(I_{2}^{(n)}\right)^{1 / 3} c\left(\theta_{2}^{(n)}\right)\right)$ and all functions are evaluated at $t$.

To prove this claim we note that $\left|c\left(\theta_{i}^{(n)}(t)\right)\right| \geqslant \rho$ if $t \in[0,2 k \pi] \backslash A_{\rho, n}, i=1,2$. Going back to Lemma $3 \cdot 2$,

$$
\begin{aligned}
\left|I_{2}^{(n)}(t)^{1 / 3} c\left(\theta_{2}^{(n)}(t)\right)\right| & \geqslant \rho\left(\frac{I_{20}^{(n)}}{4}\right)^{1 / 3} \geqslant \rho\left(\frac{\epsilon I_{10}^{(n)}}{4}\right)^{1 / 3} \geqslant \frac{\rho}{4^{2 / 3}} \epsilon^{1 / 3} I_{1}^{(n)}(t)^{1 / 3} \\
& \geqslant \frac{\rho}{4^{2 / 3}} \frac{\epsilon^{1 / 3}}{\|c\|_{\infty}}\left|I_{1}^{(n)}(t)^{1 / 3} c\left(\theta_{1}^{(n)}(t)\right)\right| .
\end{aligned}
$$

An analogous upper bound can be produced so that

$$
\epsilon^{*}=\frac{\rho \epsilon^{1 / 3}}{4^{2 / 3}\|c\|_{\infty}} .
$$

Once we know that $(3 \cdot 14)$ holds, we note that

$$
\left|x_{n}(t)\right| \geqslant \gamma \rho\left(\frac{I_{20}^{(n)}}{4}\right)^{1 / 3} \quad \text { if } t \in[0,2 k \pi] \backslash A_{\rho, n} .
$$

If we apply $(3 \cdot 2)$ on the set $\mathcal{K}_{\epsilon^{*}}$ we deduce that, for large $n$,

$$
Q\left(t ; \Theta_{0}^{(n)}, \mathcal{I}_{0}^{(n)}\right)<\frac{\delta}{4 k \pi} \quad \text { if } t \in[0,2 k \pi] \backslash A_{\rho, n} .
$$


This implies that

$$
\int_{[0,2 k \pi] \backslash A_{\rho, n}} Q\left(t ; \Theta_{0}^{(n)}, \mathcal{I}_{0}^{(n)}\right) d t<\frac{\delta}{2} .
$$

The inequalities $(3 \cdot 13)$ and $(3 \cdot 15)$ are not compatible with the assumption $(3 \cdot 11)$ and so we have reached a contradiction.

Proof of Proposition 3.4 This is now a direct consequence of the continuous dependence for linear problems. From Gronwall's Lemma we deduce that

$$
\left|\eta\left(t ; \Theta_{0}, \mathcal{I}_{0}\right)-\eta_{\infty}(t)\right| \leqslant\left(\sup _{t \in[0,2 k \pi]}\left|\eta_{\infty}\right|\right) \exp (2 k \pi L) \int_{0}^{2 k \pi}\left|\mathcal{A}\left(s ; \Theta_{0}, \mathcal{I}_{0}\right)-\mathcal{A}_{\infty}\right| d s
$$

where $\eta_{\infty}$ is the solution of

$$
\eta^{\prime}=\mathcal{A}_{\infty} \eta, \quad \eta_{1}(0)=0, \eta_{2}(0)=\mathrm{Id}
$$

and $L$ is a positive constant bounding $\left|\mathcal{A}\left(s ; \Theta_{0}, \mathcal{I}_{0}\right)\right|$ on $\mathbb{R} \times \mathbb{R}^{2} \times \mathcal{C}_{\epsilon}$, with $\left|\mathcal{I}_{0}\right|$ large. Since $\eta_{\infty 1}(t)=-\frac{\omega}{3} \operatorname{Id} t, \eta_{\infty 2}(t)=\operatorname{Id}$ the result follows from the previous lemma.

Proof of Theorem 3.1 Fix an integer $k \geqslant 1$ and numbers $\alpha, \beta \in \mathbb{R}$ with $0<\alpha<\beta$. We are going to apply the results of Section 2 to the map

$$
\left(\Theta_{0}, \mathcal{I}_{0}\right) \mapsto \Psi_{k}\left(\Theta_{0}, \mathcal{I}_{0}\right)=\left(\Theta\left(2 k \pi ; \Theta_{0}, \mathcal{I}_{0}\right), \mathcal{I}\left(2 k \pi ; \Theta_{0}, \mathcal{I}_{0}\right)\right) .
$$

First of all we observe that this map is well defined on $\left.\mathbb{R}^{2} \times\right] \widehat{l_{k}}, \infty\left[{ }^{2}\right.$ and $\Psi_{k}=\left(\Psi_{1}\right)^{k}$. This is a consequence of Lemma 3.2 and the periodicity of the equation. Moreover, also the iterates $\Psi_{h}=\left(\Psi_{1}\right)^{h}$ for $h=1, \ldots, k-1$ are well defined on $\left.\mathbb{R}^{2} \times\right] \widehat{l}_{k}, \infty\left[{ }^{2}\right.$. Finally, Proposition $2 \cdot 4$ ensures that $\Psi_{h}$, for $h=1, \ldots, k$, is an exact symplectic embedding of class $C^{1}$ satisfying $(2 \cdot 1)$ on this domain.

The final part of the proof consists in the construction, for any $\nu=\left(\nu_{1}, \nu_{2}\right) \in \mathbb{N}^{2}$ satisfying (3.4) (with $\nu^{*}$ to be chosen), of suitable open and convex subsets $\mathcal{D}(\nu) \subset$ ]$\widehat{l}_{k}, \infty\left[{ }^{2}\right.$ such that $\Psi_{k}$ satisfies the monotone twist condition on $\mathbb{R}^{2} \times \mathcal{D}(\nu)$ and

$$
-2 \pi \nu \in F\left(\Theta_{0}, \mathcal{D}(\nu)\right)
$$

for each $\Theta_{0} \in \mathbb{R}^{2}$. Here we have set, as in Section $2, F\left(\Theta_{0}, \mathcal{I}_{0}\right)=\Theta\left(2 k \pi ; \Theta_{0}, \mathcal{I}_{0}\right)-\Theta_{0}$. In the following, we also use the notation $F=\left(F_{1}, F_{2}\right)$.

For every $\nu=\left(\nu_{1}, \nu_{2}\right) \in \mathbb{N}^{2}$ define

$$
a_{i}(\nu)=\left(\frac{2 \pi \nu_{i}-1}{2 k \pi \omega}\right)^{3}, \quad b_{i}(\nu)=\left(\frac{2 \pi \nu_{i}+1}{2 k \pi \omega}\right)^{3} .
$$

Assume that $\alpha<\frac{\nu_{2}}{\nu_{1}}<\beta$. We choose $\nu_{1}+\nu_{2}$ large enough so that the rectangle

$$
\mathcal{D}(\nu)=] a_{1}(\nu), b_{1}(\nu)[\times] a_{2}(\nu), b_{2}(\nu)[\subset] 0, \infty\left[^{2} .\right.
$$

is contained in the cone $\mathcal{C}_{\varepsilon}$ with $\varepsilon=\min \left\{\frac{\alpha^{3}}{2}, \frac{1}{2 \beta^{3}}\right\}$. Then, Proposition $3 \cdot 4$ implies the existence of $\nu^{*}=\nu^{*}(k, \alpha, \beta)>0$ such that $\Psi_{k}$ satisfies the monotone twist condition on $\mathbb{R}^{2} \times \mathcal{D}(\nu)$, for every $\nu \in \mathbb{N}^{2}$ satisfying (3.4). To conclude, we just have to show that, for such $\nu$ 's and for every $\Theta_{0} \in \mathbb{R}^{2},(3 \cdot 16)$ holds true. This is a consequence of the PoincaréMiranda theorem (see [11, Generalized intermediate value th., p. 178]) for the vector 
field $\mathcal{I}_{0} \mapsto F\left(\Theta_{0}, \mathcal{I}_{0}\right)+2 \pi \nu$ on the rectangle $\mathcal{D}(\nu)$. Indeed, from Lemma $3 \cdot 3$

$$
\left\{\begin{array}{l}
F_{i}\left(\Theta_{0}, \mathcal{I}_{0}\right)+2 \pi \nu_{i}=1+O\left(\frac{1}{\nu_{i}}\right)>0 \quad \text { if } I_{i 0}=a_{i}(\nu) \\
F_{i}\left(\Theta_{0}, \mathcal{I}_{0}\right)+2 \pi \nu_{i}=-1+O\left(\frac{1}{\nu_{i}}\right)<0 \text { if } I_{i 0}=b_{i}(\nu)
\end{array} \quad i=1,2 .\right.
$$

Maybe $\nu^{*}$ has to be increased in order to get this change of sign.

Summarizing, Theorem $2 \cdot 1$ yields three geometrically different solutions of the equation

$$
\Psi_{k}\left(\Theta_{0}, \mathcal{I}_{0}\right)=\left(\Theta_{0}-2 \pi \nu, \mathcal{I}_{0}\right), \quad\left(\Theta_{0}, \mathcal{I}_{0}\right) \in \mathbb{R}^{2} \times \mathcal{D}(\nu) .
$$

Accordingly, we have three $k T$-periodic solutions $(\Theta(t), \mathcal{I}(t))$ of the Hamiltonian system (3.6) (recall that $T=2 \pi$ ) such that $\theta_{i}(k T)-\theta_{i}(0)=-2 \pi \nu_{i}$. Going back (via $(3 \cdot 5)$ ) to the system $(3 \cdot 3)$, and since $x_{i}(t)=\gamma I_{i}(t)^{1 / 3} c\left(\theta_{i}(t)\right)$, we thus find three $k T$-periodic solutions $\left(x_{1}(t), x_{2}(t)\right)$ of $(3 \cdot 3)$ such that $x_{i}(t)$ has exactly $\nu_{i}$ zeros on $[0, k T[$, for $i=1,2$.

If $k \geqslant 2$ and $\left(k, \nu_{1}, \nu_{2}\right) \in \mathbb{N}^{3}$ is visible from the origin, we can prove for such solutions the minimality of the period $k T$ in the class $\mathbb{N} T$. Indeed, suppose by contradiction that $\left(x_{1}(t), x_{2}(t)\right)$ is $l T$-periodic for some $l \in\{1, \ldots, k-1\}$; then there exist two integers $j_{1}, j_{2}$, with $j_{i} \geqslant 1$, such that $x_{i}(t)$ has exactly $j_{i}$ zeros on $[0, l T$. Moreover, the number of zeros of $x_{i}(t)$ on the interval $\left[0, k l T\left[\right.\right.$ is $j_{i} k=\nu_{i} l$. Hence, the point $\left(l, j_{1}, j_{2}\right) \in \mathbb{N}^{3}$ lies on the segment joining the origin with $\left(k, \nu_{1}, \nu_{2}\right)$, contradicting the visibility condition.

Finally, Corollary $2 \cdot 3$ implies that these subharmonics lie in different periodicity classes.

\section{REFERENCES}

[1] V. I. Arnold, Mathematical Methods of Classical Mechanics. Springer-Verlag, New YorkHeidelberg, 1978.

[2] A. Bahri and A. Berestycki, Existence of forced oscillations for some nonlinear differential equations. Comm. Pure Appl. Math. 37 (1984) 403-442.

[3] T. Ding and F. Zanolin, Periodic solutions of Duffing's equations with superquadratic potential. J. Differential Equations 97 (1992) 328-378.

[4] H. Jin, B. LiU and Y. WANG, The existence of quasiperiodic solutions for coupled Duffingtype equations. J. Math. Anal. Appl. 374 (2011) 429-441.

[5] M. Kunze and R. Ortega, Long-time stability estimates for the non-periodic Littlewood boundedness problem, Proc. London Math. Soc. 107 (2013) 39-75.

[6] M. Kunze and R. Ortega, Twist mappings with non-periodic angles. Stability and bifurcation theory for non-autonomous differential equations, Lecture Notes in Mathematics 206, Springer, 2013, 265-300.

[7] S. Lefschetz, Differential Equations: Geometric Theory. Dover Publications, Inc., New York, 1977.

[8] L. A. Lusternik and L. G. Schnirelmann, Méthodes Topologiques dans les Problèmes Variationnels. Hermann, Paris, 1934.

[9] G. R. Morris, An infinite class of periodic solutions of $x^{\prime \prime}+2 x^{3}=p(t)$. Proc. Cambridge Philos. Soc. 61 (1965) 157-164.

[10] J. Moser and E. J. Zehnder, Notes on Dynamical Systems. Courant Lecture Notes in Mathematics 12, New York University, Courant Institute of Mathematical Sciences, New York; American Mathematical Society, Providence, RI, 2005.

[11] N. Rouche and J. Mawhin, Équations différentielles ordinaires, Tome II: Stabilité et solutions périodiques. Masson et Cie, Éditeurs, Paris, 1973.

[12] S. Terracini and G. Verzini, Solutions of prescribed number of zeroes to a class of superlinear ODE's systems. NoDEA Nonlinear Differential Equations Appl. 8 (2001) $323-341$. 